\title{
La madera de cinco especies de Zanthoxylum L. (Rutaceae) con distribución en México
}

\author{
The wood of five species of Zanthoxylum L. (Rutaceae) \\ with distribution in Mexico
}

\author{
Fernanda Arenas Flores ${ }^{1}$, Agustina Rosa Andrés-Hernández ${ }^{1 *}$, \\ Teresa Terrazas ${ }^{2}$ y Carlos Castañeda 1
}

\begin{abstract}
RESUMEN
En este trabajo se describen y comparan las características anatómicas de la madera de cinco especies de Zanthoxylum. Los caracteres cualitativos y cuantitativos de la madera se analizaron a través de análisis multivariados, con la finalidad de conocer si existen caracteres que contribuyan a distinguirlas o agruparlas en las secciones reconocidas para el género. Las especies comparten los caracteres diagnósticos del género: pared de elementos de vaso $>4 \mu \mathrm{m}$, punteaduras alternas con diámetro $<6 \mu \mathrm{m}$, excepto por Z. hidalgense, fibras libriformes y parénquima marginal en bandas. Las cinco especies tienen porosidad semianular que las diferencia de otras especies de Zanthoxylum con porosidad anular y en la mayoría porosidad difusa. Se registran por primera vez para el género, drusas en vasos y fibras septadas. Los resultados del análisis de componentes principales y el fenograma mostraron que las cinco especies son entidades diferentes con una combinación de caracteres única y que los caracteres de la madera no sustentan las secciones. Zanthoxylum purpusii se caracteriza por la presencia de canales verticales traumáticos y las fibras septadas; Z. arborescens, $Z$. purpusii y Z. liebmannianum se agrupan por el parénquima marginal en bandas y los radios biseriados. Zanthoxylum hidalgense tiene los vasos más anchos, las fibras más largas y anchas y los radios mas altos y anchos, mientras que $Z$. arborescens y $Z$. liebmannianum tienen vasos más angostos y fibras más cortas y angostas. El diámetro de los vasos y la longitud y diámetro de las fibras parecen estar relacionadas con el tipo de vegetación donde las especies estudiadas se distribuyen.
\end{abstract}

PALABRAS CLAVE:

Anatomía, madera, México, porosidad semianular, Rutaceae, Zanthoxylum.

\begin{abstract}
Wood anatomy of five species of Zanthoxylum is described and compared. Qualitative and quantitative characters were analyzed through multivariate analyses to identify wood characters that permit to distinguish among species or to group them in the recognized sections within the genus. The species shared the diagnostic wood character for Zanthoxylum as of vessel wall thickness $<4 \mu \mathrm{m}$, intervessel pits $<6 \mu \mathrm{m}$, except for $Z$. hidalgense, libriform fibers, and parenchyma in marginal bands. The semiring-porosity distinguished the studied species of other Zanthoxylum species. The occurrence of druses in vessels and septate fibers are reported for the first time for the genus. The results of principal component analysis and the phenogram support the recognition of the five species studied based con a unique combination of wood characters; however, wood characteristics do not always support inclusion in the current sections. Zanthoxylum purpusii is recognized by the vertical traumatic canals
\end{abstract} México.

* $\quad$ Autor para correspondencia. c.e.: arahdm@yahoo.com.mx 
and septate fibers; Z. arborescens, Z. purpusii and $Z$. liebmannianum are grouped by parenchyma in marginal bands and biseriate rays. Zanthoxylum hidalgense is the species with the wider vessels, wider and longer fibers as well as taller and wider rays while $Z$. arborescens and $Z$. liebmannianum show the narrower vessels and shorter and narrower fibers. Vessel diameter and length and diameter of fibers appear to be related to the vegetation type where the species studied are distributed.

KEY WORDS:

Anatomy, Mexico, Rutaceae, Semiring porosity, wood, Zanthoxylum.

\section{INTRODUCCIÓN}

La familia Rutaceae es conocida como fuente de frutas cítricas y maderas satinadas, consiste en alrededor de 155 géneros y 930 especies de árboles aromáticos y arbustos (Judd et al., 2008). El género Zanthoxylum pertenece a esta familia y se ha clasificado dentro de la subfamilia Rutoideae, tribu Zanthoxyleae (Stace et al., 1993), comprende aproximadamente 200 especies, distribuidas en los trópicos de América y Asia. La mayoría de las especies de Zanthoxylum son árboles maderables, comúnmente presentan espinas en el tallo de origen floemático o del corcho (Barber, 1982); sus flores son 3,4,5-meras, epígeas, pequeñas $(3 \mathrm{~mm}-6 \mathrm{~mm}$ ) y con perianto segmentado o ligeramente diferenciado en pétalos y sépalos, pétalos de color blanco-crema o verde; los frutos son 1-5 folicular con folículos libres o ligeramente connados lateralmente; las semillas son relativamente pequeñas $(3 \mathrm{~mm}-8 \mathrm{~mm})$, de forma globosa, de color negro, brillosas y con la cicatriz funicular visible (Reynel, 1995).

Engler (1896) dividió al género en dos subgéneros Fagara y Zanthoxylum. Fagara, que se caracteriza por la presencia de perianto completo (sépalos y pétalos presentes) y lo subdividió en cuatro secciones (Macqueria, Pterota, Perijea y Tobinia). El subgénero Zanthoxylum se caracteriza por la presencia de perianto simple (sólo con tépalos). El mismo Engler (1931) reclasificó al subgénero Fagara con base en caracteres morfológicos de la flor y lo dividió en cuatro secciones que no corresponden a su primera propuesta (Blackburnia, Macqueria con tres subsecciones, Paniculatae, Perijea, PterotaMayu y Tobinia). Reynel (1995) no reconoce los subgéneros de Engler y divide al género Zanthoxylum en cinco secciones: Macqueria con una distribución Neotropical y en el Viejo Mundo, y perianto completo o incompleto con flores 4-5meras, estigmas discoidales o globosos. Novo, incluye las especies del Viejo Mundo con un perianto reducido o perianto segmentado 3-5-meras persistente en el fruto. Pterota, con una distribución Neotropical, se caracteriza por tener perianto segmentado con flores 4-meras y estigmas globosos. Tobinia, también del Neotrópico, la distingue por la presencia de flores 3-4-meras. Zanthoxylum con distribución en el Nuevo Mundo y Asia, presenta perianto segmentado indiferenciado o no, flores 3-meras, estigma globoso y ovarios con glándulas.

En cuanto a la madera de Zanthoxylum se han realizado descripciones macroscópicas y microscópicas de algunas especies de la sección Macqueria que se distribuyen en Sudamérica (Loureiro et al., 1981; Carrera y Pérez, 1988; Mendes et al., 1999; León, 2006), tienen porosidad difusa, vasos con un grosor de pared de $3.5 \mu \mathrm{m}$ a $6 \mu \mathrm{m}$, fibras no septadas $\mathrm{y}$ comúnmente parénquima en bandas; sin embargo, también se presenta el parénquima paratraqueal escaso y apotraqueal marginal. A la fecha poco se conoce de la madera de las especies de Zanthoxylum que se distribuyen en México (Bajaras- 
Morales et al., 1997). Reynel (1995) trabajó la anatomía de la madera de 61 especies y con base en sus resultados organiza seis grupos. Uno de sus grupos se caracteriza por la presencia de porosidad difusa, diámetro de las punteaduras entre $4 \mu \mathrm{m}$ y $5 \mu \mathrm{m}$, fibras de delgadas a gruesas, parénquima apotraqueal marginal en bandas o parénquima paratraqueal escaso, radios multiseriados $y$ uniseriados, que corresponden a especies de la sección Blackburnia (sensu Engler) y a Z. flavum de la sección Macqueria. En su trabajo, Reynel no integra descripciones por especie y los grupos propuestos no corresponden a ninguno de los sistemas de clasificación infragenéricos.

\section{OBJETIVO}

Describir y comparar las características anatómicas de la madera de cinco especies de Zanthoxylum que se distribuyen en México, con el propósito de conocer si existen caracteres anatómicos que contribuyan a distinguirlas y agruparlas en las diferentes secciones sensu Reynel para el género.

\section{METODOLOGÍA}

El material de estudio corresponde a muestras de madera de cinco especies pertenecientes al género Zanthoxylum, las cuales fueron recolectadas en diferentes localidades en los estados de Puebla y Querétaro (Apéndice). Las muestras se obtuvieron con un serrote, del tronco principal a una distancia $10 \mathrm{~cm}$ a $25 \mathrm{~cm}$ del suelo. Todas las muestra se fijaron en formalina-ácido acético glacialalcohol etílico 95\% por 24 h-48 h (Ruzin, 1999) y se almacenaron en una solución de glicerina-alcohol etílico 95\%-agua (1:1:1) hasta iniciar la microtecnia. Cortes transversales, tangenciales y radiales
$(20 \mu \mathrm{m})$ se hicieron con un micrótomo de deslizamiento (Leica SM 2000R). Para cada individuo, cortes sin aclarar y aclarados $(50 \%$-v/v- solución acuosa de Clorox comercial) fueron teñidos con safranina-verde rápido (Ruzin, 1999) y montados en resina sintética. Para obtener el material disociado se obtuvieron pequeñas astillas de la muestras de madera cercana al cámbium vascular y se colocaron en solución de Jeffrey (Berlyn y Miksche, 1976). Una vez disociada la madera se tiñeron en safranina acuosa $(1 \%)$ y montaron en resina sintética para obtener la longitud de los elementos de vaso y las fibras. La terminología con base en las recomendaciones de la Asociación Internacional de Anatomistas de la Madera (IAWA Committee, 1989). Se hicieron 25 mediciones para cada caracter por individuo con un microscopio Olympus BX51 y analizador de imágenes (Image ProPlus version 6.1, Media Cybernetics, 2006). Se calculó la relación If/lev, donde If es la longitud de la fibra y lev es la longitud del elemento de vaso. Los valores superiores a 2,6 indican extenso crecimiento intrusivo de la fibra (Carlquist, 2001). En las descripciones se presentan los valores de la media y en paréntesis los valores mínimo y máximo.

Para estimar la semejanza anatómica entre especies de Zanthoxylum se construyó una matriz de datos con 22 caracteres cuantitativos y cualitativos por catorce Unidades Taxonómicas Operativas (los 14 de individuos de las cinco especies), la matriz se estandarizó con unidades de desviación estándar con el objetivo de mantener el principio de ponderación entre los caracteres (Sneath y Sokal, 1973). Con esta matriz se creó una matriz de similitud mediante el coeficiente de correlación de Pearson y se generó un fenograma con el algoritmo de amalgamiento del promedio no ponderado de los pares de grupos UPGMA por 
sus siglas en inglés -unweighted pairgroup average-. A partir de la matriz estandarizada de los 22 caracteres, se obtuvo una matriz de correlación con el coeficiente de correlación Pearson y se calcularon los eigenvectores y eigenvalores, con la finalidad de conocer el porcentaje de contribución de los primeros tres componentes a la variabilidad total. Ambos análisis se realizaron con el programa NTSYS-PC versión 2.1 (Rohlf, 1998).

\section{RESULTADOS}

A continuación se presenta una descripción general y por especie, en la tabla 1 se sintetizan algunos de los caracteres cuantitativos y cualitativos para las especies, en las figuras 1-15 se ilustran las características distintivas como porosidad, inclusiones y parénquima axial y radial.

Las maderas de Zanthoxylum presentan porosidad semianular (figuras 1-5), los vasos estan arreglados en hileras radiales y solitarios; las hileras radiales van de $2-4$. Predominan los de 2 en Z. hidalgense en $63 \%$, en Z. liebmannianum en $74 \%$, en $Z$. purpusii en $87 \%$ y para Z. sp. en $73 \%$, predominan las hileras radiales de 4 en $Z$. arborescens en $86 \%$. El resto de los porcentajes para cada especie corresponde a los vasos solitarios. El porcentaje menor de vasos $/ \mathrm{mm}^{2}$ es de 7-9 para Z. hidalgense y el mayor de 24-30 para Z. arborescens. El promedio del lumen de los vasos en la madera temprana es de $33(22-48) \mu \mathrm{m}$ y en la madera tardía de 19 (8-28) $\mu \mathrm{m}$ para las especies Z. arborescens y Z. liebmannianum; para $Z$. purpusii de 40 (25-56) $\mu \mathrm{m}$ en la madera temprana y de 25 (1335) $\mu \mathrm{m}$ en la madera tardía; en $Z$. sp. de $48(31-70) \mu \mathrm{m}$ en la madera temprana y de 34 (23-50) $\mu \mathrm{m}$ en la madera tardía; los valores más altos los presenta $Z$. hidal- gense de 95 (61-124) $\mu \mathrm{m}$ y 62 (28-88) para la madera temprana y tardía respectivamente. El grosor de la pared de los vasos es semejante entre las especies, así como entre la madera temprana y tardía con un promedio mínimo de $4 \mu \mathrm{m}$ en $Z$. arborescens y máximo de $6 \mu \mathrm{m}$ en $Z$. hidalgense. La longitud de los vasos es variable de 255 (127-400) $\mu \mathrm{m}$ en Z. sp. a 386 (215-641) um en Z. purpusii (Tabla 1). Las placas de perforación son simples, las punteaduras intervasculares son alternas (figuras 6 y 7) con diámetro de 3 (2-5) $\mu \mathrm{m}$ en Z. arborescens y Z. liebmannianum, de 4(2-5) $\mu \mathrm{m}$ en $Z$. purpusii y Z. sp. y de 7(5-10) $\mu \mathrm{m}$ en Z. hidalgense; las punteaduras vaso-radio son similares a las intervasculares. Las especies presentan fibras libriformes, Z. hidalgense presenta valores mayores con 887 (2851548) $\mu \mathrm{m}$ en la longitud de fibras y de 14 (7-22) $\mu \mathrm{m}$ en el diámetro y Z. liebmannianum con los valores menores 585 (312-999) $\mu \mathrm{m}$ en la longitud de fibras y de $8(5-12) \mu \mathrm{m}$ en el diámetro de las fibras. Se muestras los valores de la media para las especies en la tabla 1.

Zanthoxylum arborescens. Los vasos sin contenidos, punteaduras con contenidos de color ámbar de naturaleza fenólica y algunas drusas (Fig. 15). Fibras libriformes no septadas, Lf/lev $=2,29$. Parénquima marginal en bandas de dos células de espesor y apotraqueal difuso; así como paratraqueal escaso (Fig. 8) y vasicéntrico; cristales prismáticos. Radios heterogéneos, 5-6 radios $/ \mathrm{mm}$, de 1-4 series (Fig. 12), en su mayoría biseriados altura de 273 (110-556) $\mu \mathrm{m}$ y 11 (4-30) células en altura, anchura de 31 (8-60) $\mu \mathrm{m}$, las células erectas en los márgenes de los radios; contenidos obscuros y cristales prismáticos en algunas células.

Zanthoxylum hidalgense. Los vasos y punteaduras sin contenidos de naturaleza fenólica o mineral. Fibras libriformes no septadas, Lf/lev $=3,04$. 


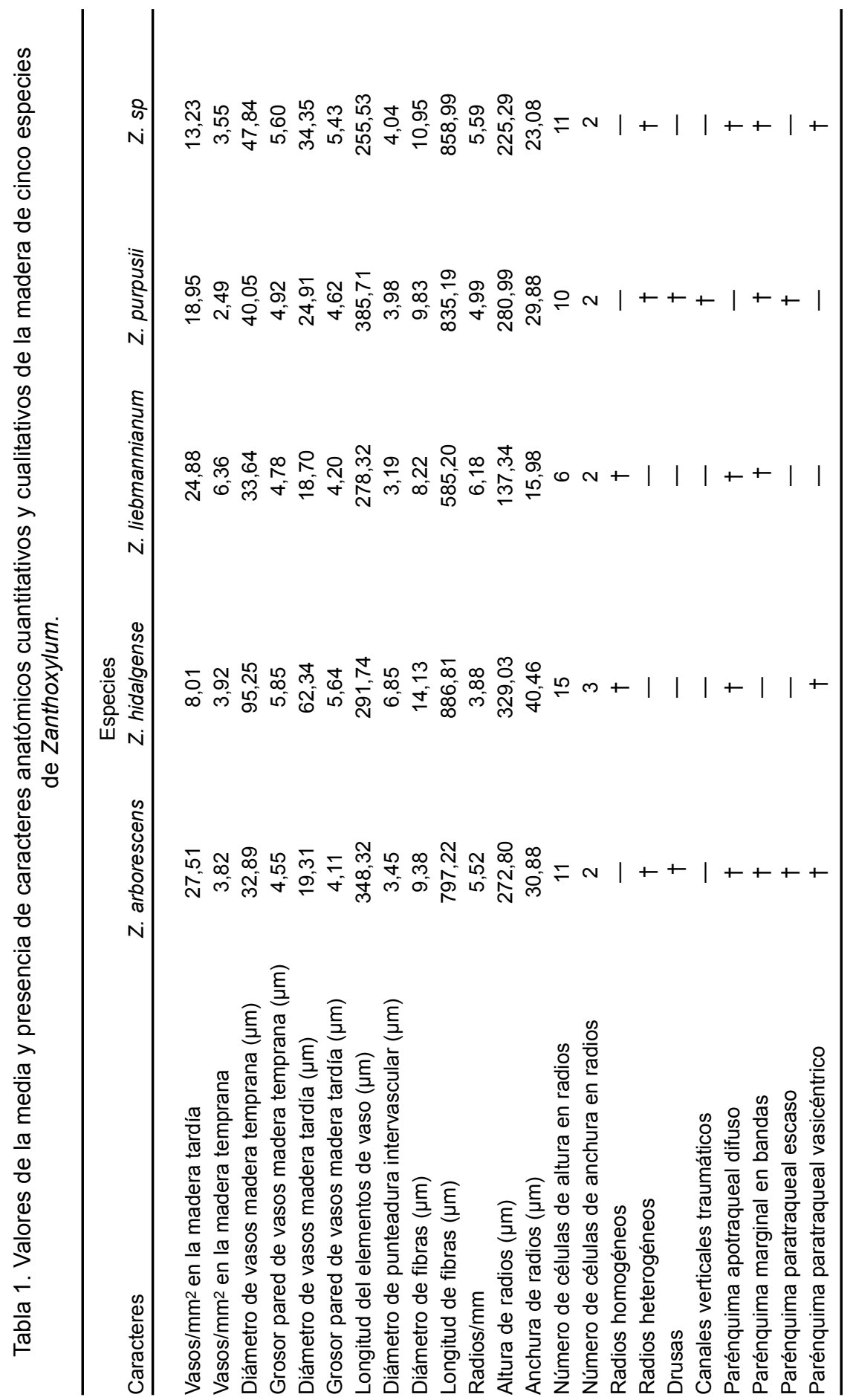



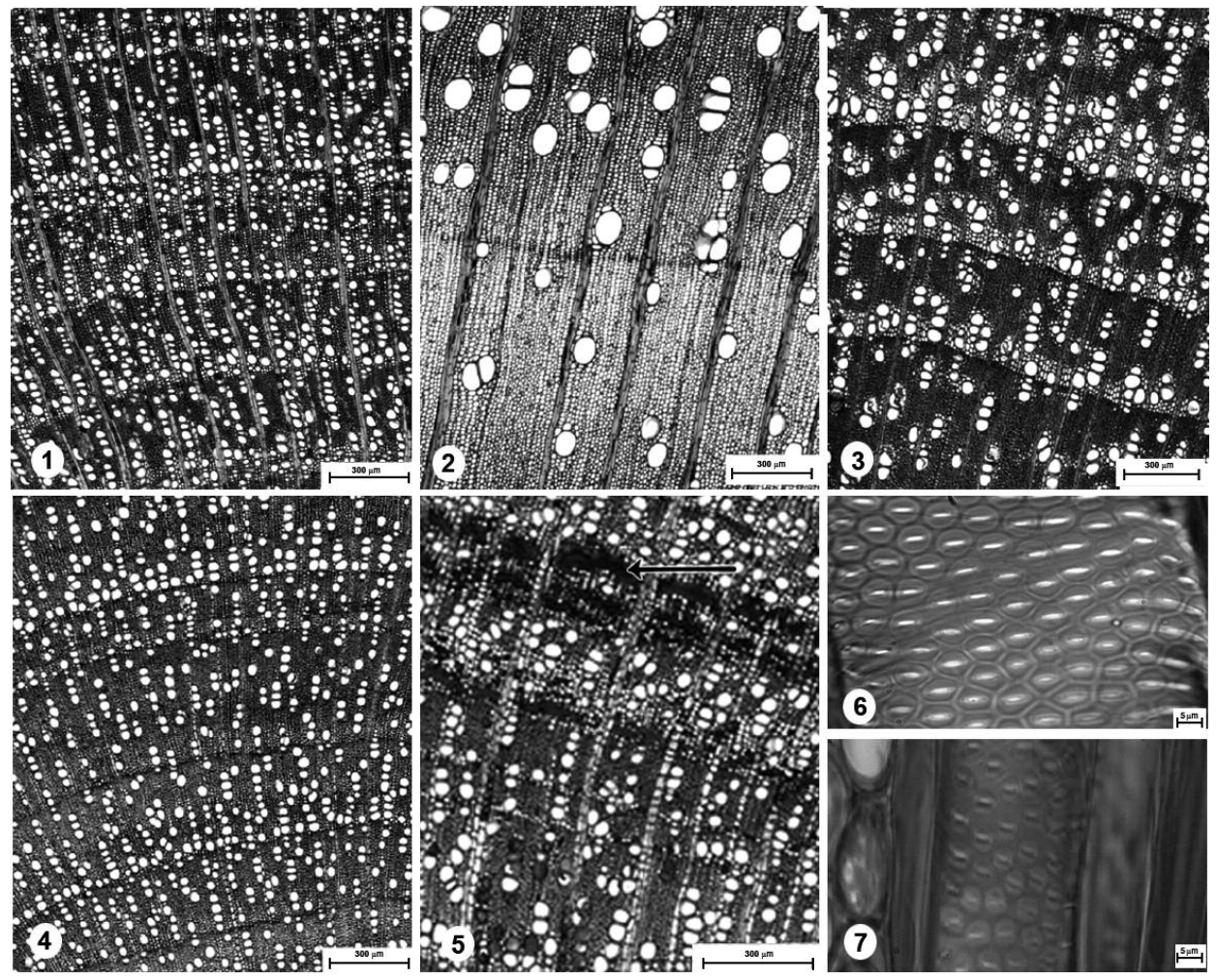

Figuras 1-7. Porosidad semianular y elementos de vasos en Zanthoxylum.

1. vasos en hileras radiales (2-4) predominan los de 4, en la madera tardía,

Z. arborescens; 2 . vasos en grupos escasos, predominan las hileras radiales de 2 ,

Z. hidalgense; 3. vasos en arreglo dendrítico en madera tardía, Z. $s p$.;

4. vasos en hileras radiales, $Z$. liebmannianum y 5 . canales traumáticos, $Z$. purpusii.

6. Punteaduras intervasculares alternas $>5 \mu \mathrm{m}, Z$. hidalgense.

7. Punteaduras intervasculares alternas $<5 \mu \mathrm{m}$, Z.arborescens. 

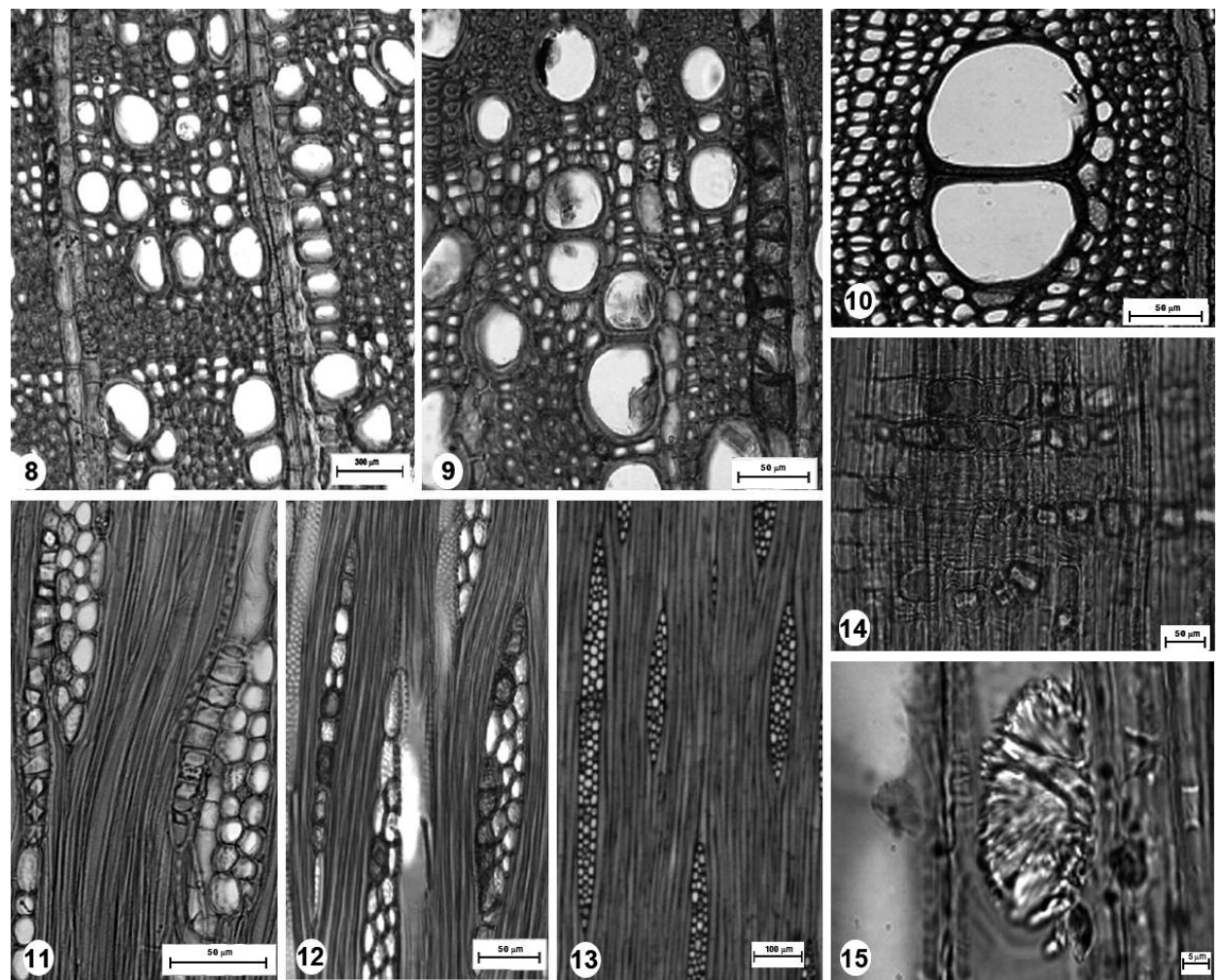

Figuras 8-15. Parénquima axial y radial en Zanthoxylum. 8. Parénquima paratraqueal escaso, Z. arborescens; 9. Parénquima marginal en bandas y paratraqueal escaso,

Z. purpusii; 10. Parénquima paratraqueal vasicéntrico, Z. hidalgense;

11. Serie parenquimatosa con cristales prismáticos, $Z$. sp.;

12. Radios uni, bi y triseriados, Z. arborescens; 13. Radios triseriados, Z. hidalgense;

14. Radios heterogéneos con cristales prismáticos, $Z$. liebmannianum;

15. Drusa en elemento de vaso, $Z$. arborescens. 
Parénquima apotraqueal difuso y paratraqueal vasicéntrico (Fig. 10). Radios homogéneos, 3-4 radios $/ \mathrm{mm}$, de 1-6 series, en su mayoría triseriados (Fig. 13), con una altura de 329 (80-703) $\mu \mathrm{m}$ y 14 (3-31) células de altura, con anchura de 40 (10-69) $\mu \mathrm{m}$; contenidos de color obscuro y cristales prismáticos en algunas células.

Zanthoxylum liebmannianum. Los vasos sin contenidos y punteaduras ocasionalmente contenidos de color ámbar y drusas. Fibras libriformes no septadas, $\mathrm{Lf} / \mathrm{lev}=2,10$. Parénquima marginal en bandas compuesto de una sola célula de espesor y apotraqueal difuso, así como paratraqueal escaso; con cristales prismáticos en algunas células. Radios homogéneos, 5-7 $\mathrm{radios} / \mathrm{mm}$, de 1-3 series en su mayoría biseriados, altura de 137 (73-296) $\mu \mathrm{m}, 7$ (3-16) células de altura, anchura de 16 (5-31) $\mu \mathrm{m}$, contenidos de color obscuro y cristales prismáticos en algunas células (Fig. 14).

Zanthoxylum purpusii. Los vasos sin contenidos color ámbar, pero con algunas drusas. Fibras libriformes septadas, Lf/lev $=2,29$. Parénquima marginal en bandas de 3 a 4 células de espesor y paratraqueal escaso (Fig. 9) con cristales prismáticos; canales verticales traumáticos (Fig. 5). Radios heterogéneos, 4-5 radios $/ \mathrm{mm}$, de 1-4 series, en su mayoría biseriados, altura de $280(90-620) \mu \mathrm{m}, 10$ (2-24) células de altura, anchura de 30 (4-61) $\mu \mathrm{m}$, las células erectas en los márgenes de los radios; contenidos de color obscuro y cristales prismáticos en algunas células.

Zanthoxylum sp. Los vasos con tendencia a arreglo dendrítico (Fig. 3), contenidos color ámbar en algunos vasos. Fibras libriformes no septadas, $\mathrm{Lf} / \mathrm{lev}=3,24$. Parénquima marginal en bandas de dos células de espesor y apotraqueal difuso, así como paratra- queal vasicéntrico; abundantes cristales prismáticos (Fig. 11). Radios heterogéneos, 5-6 radios/mm, de 1-3 series en su mayoría biseriados (Fig. 11), altura de 225 (66-547) $\mu \mathrm{m}, 11$ (2-29) células de altura, anchura de $23(11-34) \mu \mathrm{m}$; las células erectas en los márgenes de los radios; cristales prismáticos en algunas células.

Análisis multivariados. El análisis de componentes principales reveló que tres componentes explican cerca de $95 \%$ de la varianza y los caracteres que tuvieron mayor peso fueron diámetro de vasos y grosor de pared de vasos en madera temprana y tardía, diámetro de punteaduras intervasculares y diámetro de fibras (Tabla 2). Estos caracteres junto con los caracteres cualitativos permitieron distinguir a las cinco especies de Zanthoxylum (Fig. 16). En el fenograma se recuperan dos grupos con base en la similitud de su madera (Fig. 17). En el grupo A están Z. hidalgense y Z. sp. a una distancia de 0,24 . En el grupo $B$ grupo se recuperan las especies Z $Z$. arborescens y Z. purpusii, estas especies se relacionan a una distancia de 0,7 , considerando a estas dos especies como las más parecidas anatómicamente, entre estas especies las distancias no son significativas, pero la ubicación entre ellas en el fenograma (Fig. 17) es separada y Z. liebmannianum se une a estas dos especies a una distancia de 0,13.

\section{DISCUSIÓN}

La madera de las cinco especies de Zanthoxylum comparten las características anatómicas, grosor de la pared de los vasos $(4,11 \mu \mathrm{m}-5,65 \mu \mathrm{m})$, punteaduras alternas pequeñas $(<5 \mu \mathrm{m}$ de diámetro), fibras libriformes y parénquima en bandas. Estos caracteres ya se habían considerado como diagnósticos para la madera del género (Loureiro et al., 1981; 


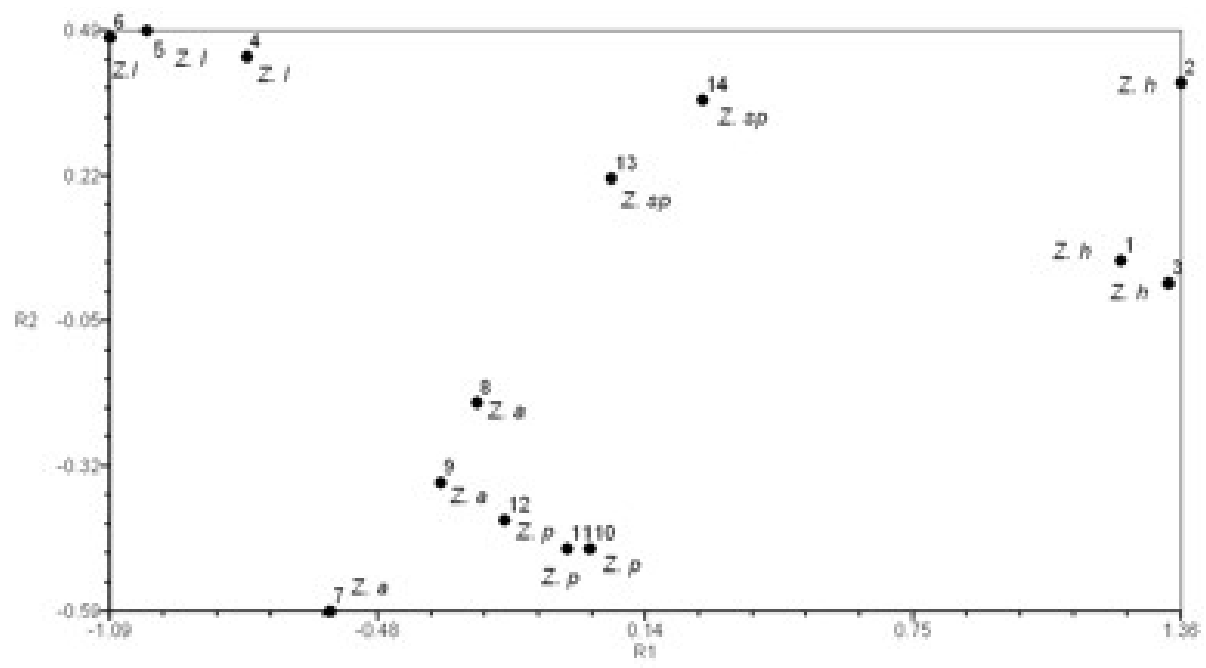

Figura 16. Arreglo espacial de las cinco especies de Zanthoxylum generado a través del análisis de Componentes Principales con base los caracteres de la tabla 2.

$\mathrm{R} 1$ = componente principal uno, R2 = componente principal dos, Z. $a=Z$. arborescens, Z. $\mathrm{h}=$ Z. hidalgense, Z. I = Z. liebmannianum, Z. $\mathrm{p}=$ Z. purpusii, Z. $\mathrm{p}=$ Z. $\mathrm{sp}$.

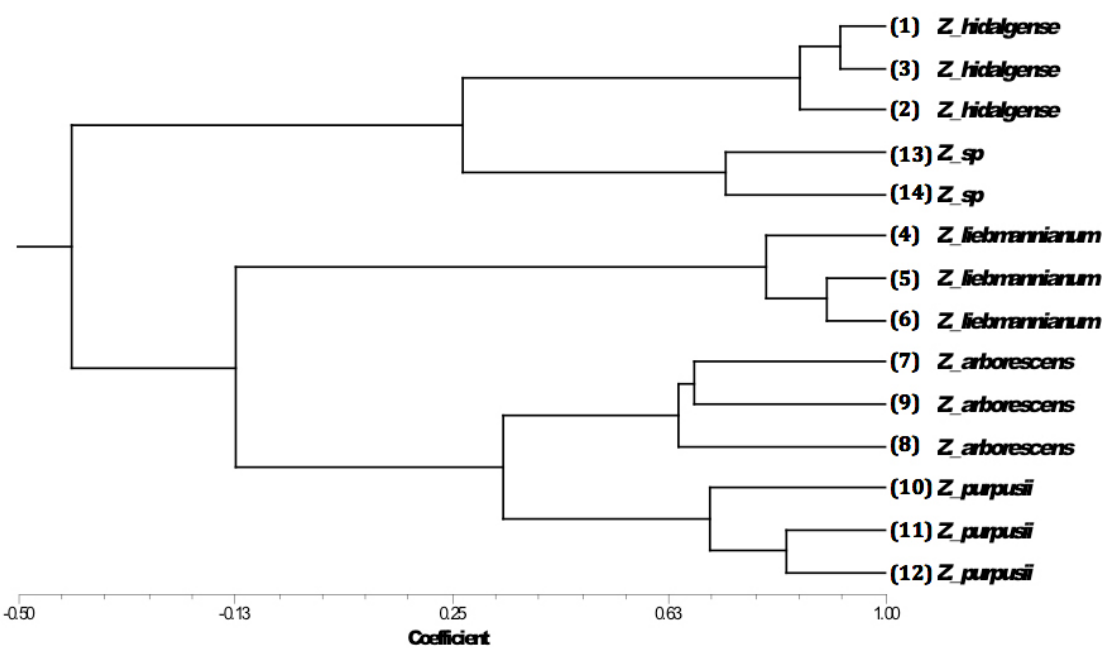

Figura 17. Fenograma para 14 individuos de las cinco especies de Zanthoxylum estudiadas, generado con el coeficiente de correlación y el método de agrupamiento UPGMA (por sus siglas en inglés). 
Tabla 2. Valores de los eigen-vectores para los tres primeros componentes de los caracteres de la madera de las cinco especies de Zanthoxylum.

Las variables con valores más altos se indican cono asterisco.

\begin{tabular}{|c|c|c|c|}
\hline \multirow[b]{2}{*}{ Caracteres } & \multicolumn{3}{|c|}{ eingvectores } \\
\hline & Componente 1 & Componente 2 & Componente 3 \\
\hline Vasos $/ \mathrm{mm}^{2}$ en la madera tardía & $-0,926$ & $-0,087$ & 0,232 \\
\hline Vasos $/ \mathrm{mm}^{2}$ en la madera temprana & $-0,319$ & 0,834 & 0,442 \\
\hline Diámetro de vasos, madera temprana & $0,966^{*}$ & 0,626 & 0,239 \\
\hline Grosor pared de vasos, madera temprana & $0,918^{*}$ & 0,247 & $-0,264$ \\
\hline Diámetro de vasos, madera tardía & $0,988^{*}$ & 0,067 & 0,118 \\
\hline rosor pared de vasos, madera tardía & $0,915^{\star}$ & 0,137 & $-0,347$ \\
\hline Longitud de elementos de vaso $(\mu \mathrm{m})$ & $-3,111$ & $-0,903^{*}$ & 0,246 \\
\hline Diámetro de punteaduras $(\mu \mathrm{m})$ & $0,961^{*}$ & $-0,055$ & 0,254 \\
\hline Diámetro de fibras $(\mu \mathrm{m})$ & $0,995^{\star}$ & $-0,079$ & 0,040 \\
\hline Longitud de fibras $(\mu \mathrm{m})$ & 0,718 & $-0,571$ & $-0,379$ \\
\hline Radios/mm & $-0,870$ & 0,401 & $-0,258$ \\
\hline Altura de radios $(\mu \mathrm{m})$ & 0,685 & $-0,709$ & 0,099 \\
\hline Anchura de radios $(\mu \mathrm{m})$ & 0,741 & $-0,585$ & 0,285 \\
\hline Número de células de altura en radios & $0,925^{\star}$ & $-0,264$ & $-0,068$ \\
\hline Número de células de anchura en radios & 0,896 & $-0,223$ & 0,329 \\
\hline Tipo de radios & $-0,300$ & $-0,628$ & $-0,685^{*}$ \\
\hline Drusas & $-0,440$ & $-0,888$ & 0,069 \\
\hline Canales verticales traumáticos & $-0,204$ & $-0,683$ & 0,094 \\
\hline Parénquima apotraqueal difuso & 0,440 & 0,888 & $-0,069$ \\
\hline Parénquima marginal en bandas & $-0,890$ & $-0,052$ & $-0,451$ \\
\hline Parénquima paratraqueal escaso & $-0,867$ & $-0,301$ & 0,386 \\
\hline Parénquima paratraqueal vasicéntrico & 0,867 & 0,301 & $-0,386$ \\
\hline
\end{tabular}

Carrera y Pérez, 1988; Mendes et al., 1999; León, 2006). Cabe mencionar que Z. hidalgense presenta punteaduras de $6,85 \mu \mathrm{m}$, valor superior a la amplitud reportada para las otras especies del género previamente estudiadas. Así, también otros autores registran para los elementos de vaso una longitud que fluctúa entre $46 \mu \mathrm{m}$ y $200 \mu \mathrm{m}$ (Loureiro et al., 1981; Carrera y Pérez, 1988; Mendes et al., 1999; León, 2006). Sin embargo, las cinco especies estudiadas presentan un valor de la media superior para este caracter, (255-385) $\mu \mathrm{m}$ (Tabla 1). BajarasMorales et al. (1997) encontraron este mismo rango en la longitud de los vasos para Z. kellermanii. Asimismo, la longitud de las fibras es mayor para las especies estudiadas en este trabajo.

El género se caracteriza por su porosidad difusa, ya que de 70 especies descritas sólo cinco presentan porosidad anular o semianular (Loureiro et al., 1981; Carrera y Pérez, 1988; Reynel, 1995; Bajaras-Morales et al., 1997; Mendes et al., 1999; InsideWood, 2004; León, 2006), incrementándose a 10 con las especies estudiadas en este trabajo. La densidad de vasos solitarios en la madera temprana fue más abundante en $Z$. liebmannianum $\left(6 / \mathrm{mm}^{2}\right)$ que en las otras cuatro especies estudiadas y la menor densidad de vasos en la madera tardía se encontró en 
Z. hidalgense $\left(8 / \mathrm{mm}^{2}\right)$, considerándose escasos en comparación con especies de otras familias (Terrazas, 1994). Los radios heterogéneos son comunes en Zanthoxylum (Loureiro et al., 1981; Carrera y Pérez, 1988; Reynel, 1995; BajarasMorales et al., 1997; Mendes et al., 1999; InsideWood, 2004; León, 2006), aunque se presentan también homogéneos como en Z. hidalgense y en Z. liebmannianum, este tipo de radios únicamente se habían registrado en otras tres especies del género (InsideWood, 2004). El género se caracteriza por presentar fibras no septadas (Reynel, 1995; InsideWood, 2004). En este trabajo, por primera vez, se registran fibras septadas en $Z$. purpusii. Aguilar-Rodríguez y Terrazas (2001), señalan que las fibras no septadas pueden mantener protoplasto y núcleos vivos con la finalidad de almacenar material de reserva y ser usado en el momento de reiniciar la actividad cambial. Por otro lado también las fibras septadas se consideran como un tipo de células de almacenamiento y los septos son indicadores de longevidad (Carlquist, 1988, 1997). Consideramos que ambos tipos de fibras pueden estar relacionadas con la porosidad semianular de dichas especies y presencia de septos en las fibras de Z. purpusii podría ser considerada como una novedad evolutiva.

Los cristales prismáticos están presentes en todas las especies del género (Loureiro et al., 1981; Carrera y Pérez, 1988; Reynel, 1995; Mendes et al., 1999; León, 2006). Los cristales son comunes en la madera de las angiospermas en células radiales, parénquima axial, a veces en fibras (Metcalfe y Chalk, 1983; Carlquist, 1989; Franceschi y Nakata, 2005); la presencia de drusas es rara en los vasos (Espinoza de Pernía, 1987) como ocurre con Z. arborescens y Z. purpusii. La presencia de estos contenidos está asociada con varias funciones de la planta o con la edad del individuo, así como con el clima y tipo de suelo (Aguilar-Rodríguez y Terrazas, 2001; Franceschi y Nakata, 2005). La presencia de drusas en estas dos especies se puede atribuir al tipo de suelo, ya que ambas especies se colectaron en la misma zona edáfica.

Con respecto al parénquima, el más común fue en bandas, presente en cuatro de las especies estudiadas. Sin embargo, se registraron otros tipos de parénquima, apotraqueal difuso, paratraqueal escaso y vasicéntrico, siendo $Z$. arborescens la especie con mayor diversidad al observarse todos los tipos de parénquima. Esto se encuentra asociado a caracteres evolucionados en las angiospermas (León y Espinoza de Pernía, 1999).

El fenograma y el diagrama de dos dimensiones del análisis de componentes principales mostraron que los individuos de cada especie se agrupan dentro de su respectiva especie con base en la combinación de caracteres de su madera. Zanthoxylum purpusii, además se distingue por la presencia de canales traumáticos y sus fibras septadas. Además, el fenograma mostró mayor similitud en la madera entre especies de diferentes secciones que entre secciones. Por ejemplo, en Z. arborescens (Sección Macqueria) y Z. purpusii (Sección Zanthoxylum) tienen radios heterogéneos, parénquima paratraqueal escaso y drusas en los vasos. Aunque en el análisis de componentes principales no sea clara la separación de Z. arborescens (9) y Z. purpusii (12), en el fenograma (Fig. 17) se demuestra la identidad específica entre ellas. Estas dos especies se agrupan con Z. liebmannianum (Sección Pterota), la presencia de parénquima marginal en bandas y radios biseriados. La especie más disímil de las demás fue $Z$. hidalgense (Sección Macqueria), la cual no tiene parénquima marginal en bandas $y$ presenta valores altos para diámetro de 
vasos en madera temprana y tardía (95,25 $\mu \mathrm{m}$ y $62,34 \mu \mathrm{m})$, grosor de la pared de vasos en madera temprana y tardía $(5,85$ $\mu \mathrm{m}$ y $5,64 \mu \mathrm{m})$, diámetro de punteaduras $(6,85 \mu \mathrm{m})$, diámetro y longitud de fibras $(14,13 \mu \mathrm{m}$ y $886,81 \mu \mathrm{m})$, y altura y anchura de radios $(329,03 \mu \mathrm{m}$ y $40,46 \mu \mathrm{m})$. Los grupos revelados en el fenograma tienen muy pocos caracteres únicos que los distinguen, por lo que el estudio de un mayor número de especies de Zanthoxylum presentes en México permitirá confirmar la existencia de dos grupos.

Las especies de Zanthoxylum presentes en México se distribuyen en diferentes tipos de vegetación y algunas de sus características anatómicas parecen ser adaptaciones a dichos ambientes, como lo sugieren el diámetro y arreglo de los vasos, así como la longitud y diámetro de las fibras. Zanthoxylum hidalgense se distribuye en el bosque templado y presenta los vasos de mayor diámetro con fibras más largas y de mayor diámetro. Estos valores son similares a Z. kellermanii, que se distribuye en una selva alta perennifolia pero tiene porosidad difusa; mientras que $Z$. arborescens y Z. liebmannianum, que se distribuyen en el matorral xerófilo, presentan el menor diámetro de vasos y de longitud y diámetro de fibras y $Z$. purpusii, que se colectó en bosque tropical caducifolio, presenta valores intermedios. Esta última especie, junto con la especie no determinada, tienen la mayor abundancia de parénquima marginal y vasos arreglados en grupos o patrón dendrítico. Carlquist (2001) sugiere que valores del Lf/lev superiores a 2,6 indican especialización hacia ambiente xéricos. Comparado con otras familias, Lf/lev es alto para las cinco especies estudiadas. Sin embargo, una mayor especialización hacia el crecimiento intrusivo se encontró en Zanthoxylum hidalgense y en la especie no identificada.
Se concluye que las cinco especies de Zanthoxylum estudiadas que se distribuyen en México comparten los caracteres de la madera ya señalados por otros autores que las reconocen dentro del género. Sin embargo, se encontraron caracteres como la presencia de drusas en algunos vasos y las fibras septadas que se registran por primera vez para el género, sugiriendo la necesidad de estudiar un mayor número de especies. La porosidad semianular y el diámetro de los vasos entre otros caracteres parecen ser adaptaciones a los ambientes estacionales (templados $o$ áridos) donde se distribuyen las especies de Zanthoxylum en México.

\section{AGRADECIMIENTOS}

A la doctora Sombra Patricia Rivas Arancibia, por los análisis estadísticos (Escuela de Biología, BUAP). A los revisores por sus comentarios, en especial al doctor Raymundo Dávalos por su revisión crítica. Al laboratorio de fanerógamas del Instituto de Biología de la UNAM. Al financiamiento PROMEP/1035/08/1641 para el desarrollo del proyecto y por la beca otorgada a la primera autora.

\section{REFERENCIAS}

Aguilar-Rodríguez, S. y T. Terrazas. 2001. Anatomía de la madera de Buddleja L. (Buddlejaceae): análisis fenético. Madera y Bosques 7(2):63-85.

Barajas-Morales, J., A.G. Ángeles y S.P. Solís. 1997. Anatomía de maderas de México: Especies de una selva alta perennifolia. I. Instituto de Biología. Universidad Nacional Autónoma de México. México. $125 \mathrm{p}$.

Barber, C. 1982. On the nature and development of the corky excrescences 
on stems of Zanthoxylum. Annals of Botany 6:155-166.

Berlyn, G.P. y J.P. Miksche. 1976. Botanical microtechnique and cytochemistry. The lowa State University Press. Ames, lowa. 326 p.

Carrera, R. y E. Pérez. 1988. Descripción anatómica de la madera de ocho especies forestales. Revista Forestal Baracoa 18(1):17-37.

Carlquist, S. 1988. Comparative wood anatomy. Systematic, ecological and evolutionary aspects of dicotyledon wood. Springer-Verlag, Berlin. 436 p.

Carlquist, S. 1989. Wood Comparative Anatomy. Springer-Verlag. Nueva York.

Carlquist. S. 1997. Wood Anatomy of Buddlejaceae. Aliso 15(1):41-56.

Carlquist, S. 2001. Comparative wood anatomy. Springer-Verlag, Berlin. $448 \mathrm{p}$.

Engler, A. 1896. Rutaceae. Nat. Pflanzenfam. III. 4:95-201.

Engler, A. 1931. Rutaceae. In A. Engler y K. Prantl (Eds.). Die Naturlichen Pflanzenfamilien, 2a. ed., vol. 19a. Engelmann Leipzing. p: 187-359.

Espinoza de Pernía, N. 1987. Cristales y sílice en la madera de dicotiledóneas de Latinoamérica. Revista Pittieria 15:13-65.

León H. y Espinoza de Pernía. 1999. Parénquima radial disyuntivo y filogenia. Revista Forestal Venezolana 43(2):137-146.

Franceschi, V.R. y P.A. Nakata. 2005. Calcium oxalate in crystals in plants: formation and function. Annual Review of Plant Biology 56:41-71.
IAWA Committee. 1989. List of microscopic features for hardwood identification. IAWA Bulletin new series 10:219-332.

InsideWood. 2004-onwards. En línea www.insidewood.lib.ncsu.edu /search. [07-01-2009].

Judd, W.S., C.S. Campbell, E.A. Kellogg, P.F. Stevens y M.J. Donoghue. 2008. Plant systematics. A phylogenetic approach. 3a. ed. Sinauer Associates. Masachusetts. $611 \mathrm{p}$.

León, H. 2006. Anatomía de la madera de 13 especies del Orden Sapindales que crecen en el Estado de Mérida, Venezuela. Acta Botánica Venezolana 29:269-296.

Loureiro, A.A., J.F. Vasconcelos y W.P. Albuquerque. 1981. Anatomia do lenho de 4 espécies de Zanthoxylum Linnaeus (Rutaceae) da Amazonia. Acta Amazonica 11(4):809-820.

Mendes, L.M., R.M. Silva, P.F. Trugilho y G.A. López. 1999. Anatomia e características Fisico-químicas da madeira de Zanthoxylum tingoassuiba St. Hil. de ocorrência na Região de Lavras/M.G. CERNE 5(1):15-25.

Metcalfe, C.R. y L. Chalk. 1983. Anatomy of the Dicotyledons, vol. II. 2a. ed. Clarendon Press. Oxford. 297 p.

Reynel, C. 1995. Systematics of Neotropical Zanthoxylum (Rutaceae) with an account on the wood anatomy of the genus. Ph.D. thesis, University of Missouri, St. Louis. 576 p.

Rohlf, F.J. 1998. NTSYS-pc numerical taxonomy and multivariate analysis system. Release 2.02 Exeter Software, Setauket. Nueva York. 
Ruzin, E. 1999. Plant microtechnique and microscopy, Oxford University Press. Nueva York. 322 p.

Stace, H.H., J.A. Armstrong y S.H. James. 1993. Cytoevolutionary patterns in Rutaceae. Plant Systematics and Evolution 187: 1-28.

Sneath P.H y R.R. Sokal. 1973. Numerical taxonomy. The principles and practices of numerical classification. W.H. Freeman and Company, San Francisco. $573 \mathrm{p}$.

Terrazas, S.T. 1994. Wood anatomy of the Anacardiaceae: ecological and phylogenetic interpretation. Tesis doctoral. University of North Carolina of Chapel Hill. 321 p.

\section{APÉNDICE}

\section{Especies y secciones de Zanthoxylum estudiadas, así como su localización geográfica}

Z. arborescens Rose. Sección Macqueria. Puebla: $350 \mathrm{~m}$ antes de entrar a Chila de las Flores, Acatlán, 17²57'27.1'"9752'03.2", 1726 msnm, Arenas-Flores 4, 5, 6, 7 (MEXU). Z. hidalgense Lundell, Sección Macqueria. Querétaro: Mpio. Landa, camino hacia Agua Zarca a $6 \mathrm{~km}$ del entronque del Lobo, 2116'24.9"'9906'29.3", 1850 msnm, Arenas-Flores 15, 16, 17, 18, 19 (MEXU). Z. liebmannianum (Engl.) P. Wilson, Sección Pterota. Puebla: junto a la estación de microondas carretera TecamachalcoTehuacán, 18³4'45.7'-97³4'50.0"', 1879 msnm, Arenas-Flores 1, 2, 3 (MEXU). Z. purpusii Brandegee, Sección Zanthoxylum. Puebla: $5 \mathrm{~km}$, saliendo de Acatlán hacia Izúcar de Matamoros, 18¹1'43.6"9808'30.1", 1750 msnm, Arenas-Flores 8, 9, 10 (MEXU). Z. sp. Puebla: cerro del Tenzo, antes de San Juan Atzompa, 1844'6.3"'-9758'0.9", 1909 msnm, Andrés-Hernández 111, 112 (MEXU).

Manuscrito recibido el 11 de agosto de 2010.

Aceptado el 1 de diciembre de 2011.

Este documento se debe citar como: Arenas Flores, F., A.R. Andrés-Hernández, T. Terrazas y C. Castañeda. La madera de cinco especies de Zanthoxylum L. (Rutaceae) con distribución en México. Madera y Bosques 18(1):43-56. 